\title{
Epibiontes en juveniles de tortugas carey Eretmochelys imbricata varadas en la costa del Departamento de Rocha, Uruguay
}

\author{
Epibiota of juvenile Hawksbill Sea Turtles Eretmochelys imbricata stranded \\ in the coast of Rocha Department, Uruguay

\section{Catalina Velasco-Charpentier ${ }^{1 *}$, Felipe Pizarro-Mora ${ }^{1}$, Andrés Estrades ${ }^{2}$ y Gabriela M. Veléz-Rubio ${ }^{2,3}$}

\author{
${ }^{1}$ Facultad de Ciencias del Mar y de Recursos Naturales, Universidad de Valparaíso, Casilla 5080, Viña del Mar, Chile.*cata.velasco91@ gmail.com \\ ${ }^{2}$ ONG Karumbé, Av. Rivera 3245, Montevideo, CP 11400, Uruguay \\ ${ }^{3}$ Instituto Cavanilles de Biodiversidad y Biología Evolutiva, Universidad de Valencia, Aptdo. 22085, E-46071 Valencia, España
}

\begin{abstract}
The hawksbill turtle (Eretmochelys imbricata) is the most threatened sea turtle species in the world. An important aspect of the biology of sea turtles is the study of colonizing fauna, i.e., their epibiota. The aim of this study is a taxonomic determination on the epibiota found on 4 hawksbills turtles stranded in 2009 and 2011 on the coast of Rocha Department, Uruguay. The epibiota was composed by algae from the class Phaeophyceae (Sphacelaria sp. and Hincksia mitchelliae) and invertebrates from the classes Cirripedia (Platylepas hexastylos, Chelonibia testudinaria and Amphibalanus improvisus) and Hirudinea (Ozobranchus margoi), with greater predominance of $P$. hexastylos cirripeds $(n=365)$, a result that is consistent with other similar studies. Hincksia mitchelliae and A. improvisus are new reports as hawksbill turtles' epibiota.
\end{abstract}

Key words: Cheloniidae, barnacles, Southwestern Atlantic

\section{INTRODUCCIÓN}

La tortuga carey, Eretmochelys imbricata (Linnaeus, 1766), es una especie migratoria que presenta una distribución circunglobal en aguas tropicales y, en menor medida, en aguas subtropicales (Mortimer \& Donnelly 2008). En sus primeros años de vida, esta especie habita en el ambiente oceánico, donde se alimenta de organismos epipelágicos (Blumenthal et al. 2009) y posteriormente, los juveniles reclutan en zonas de alimentación costera cuando alcanzan longitudes entre 19,5 y $30,0 \mathrm{~cm}$ de largo recto de caparazón (para la zona del Caribe, Butrago \& Guada 2002). Los juveniles tardíos y adultos se vinculan estrechamente con los arrecifes coralinos y fondos rocosos (Meylan \& Donnelly 1999). De las 7 especies de tortugas marinas, la tortuga carey es la que sufre un mayor riesgo de extinción a nivel mundial; actualmente está incluida en la categoría 'Críticamente Amenazada' por la Unión Internacional para la Conservación de la Naturaleza (UICN 2014).

La costa uruguaya constituye una importante área de alimentación y corredor migratorio entre distintas zonas del Atlántico Sudoccidental (ASO) para individuos juveniles de tortuga verde (Chelonia mydas), juveniles tardíos y adultos de tortugas cabezona (Caretta caretta) y tortugas laúd (Dermochelys coriacea), mientras que la tortuga olivácea (Lepidochelys olivacea) y carey han sido registradas esporádicamente (Vélez-Rubio et al. 2013). En Uruguay se han reportado hasta la fecha un total de 10 tortugas carey (Estrades et al. 2013, Vélez-Rubio et al. 2013), con un único reporte más al sur, en Bahía de Samborombón, Argentina ${ }^{1}$. La presencia de esta especie es poco común en zonas templadas del ASO, ya que su principal área de distribución se localiza entre Ceará y Sao Paulo, Brasil (Marcovaldi et al. 2007).

Un aspecto importante del estudio de la biología de las tortugas marinas es el conocimiento de su fauna acompañante y colonizadora, es decir, su epibiota. Se define como epibionte cualquier organismo que vive y crece sobre otro ser vivo, siendo el basibionte el organismo que funciona como sustrato en esta asociación (Liria 2011). Existe una gran cantidad de organismos capaces de colonizar la superficie de las tortugas marinas (Schärer 2003, Liria 2011), ya que éstas ofrecen un sustrato para el asentamiento que es temporalmente estable y adecuado a la escala de duración del ciclo de vida de los epibiontes (Domènech et al. 2014). La relación epibionte-tortuga puede resultar muy estrecha y compleja e incluso específica de especie; existen organismos que sólo se encuentran asociados a tortugas marinas (Hernández-Vázquez \& Valadez-González 1998).

Los datos de epibiota para tortuga carey en Uruguay eran

${ }^{1}$ Bruno I \& D Albareda. 2009. Primer registro de la tortuga carey Eretmochelys imbricata (Linnaeus, 1766) en Argentina. IV Jornadas de Investigación y Conservación de Tortugas Marinas del ASO, Libro de resúmenes 30 de septiembre-1 de octubre de 2009, Mar del Plata, Argentina, p. 147. 
inexistentes, siendo el objetivo del presente estudio analizar y describir por primera vez los epibiontes presentes en tortugas carey juveniles varadas en la costa uruguaya. Además de su valor intrínseco, el conocimiento de la composición taxonómica, los patrones de distribución y la abundancia de los epibiontes representa un elemento útil para entender mejor la relación basibionte-epibionte y ayudar así a ampliar el conocimiento biológico y ecológico de una especie amenazada.

\section{Materiales y MÉTODOS}

La costa uruguaya abarca 710 km (Fig. 1) y se encuentra bajo la influencia principal de la corriente de Malvinas durante otoño e invierno y por la corriente de Brasil durante primavera y verano, determinando una gran amplitud anual tanto en las temperaturas del agua como en su salinidad (Ortega \& Martínez 2007). Para determinar la epibiota de la tortuga carey se analizaron 4 ejemplares de tortugas juveniles que vararon vivas, 1 en 2009 y 3 en 2011. Los ejemplares fueron reportaron a través de censos de varamientos de tortugas marinas en playa o por la Red de Rescate y Varamientos de Tortugas Marinas de Uruguay (RRVTM), coordinada por la ONG Karumbé (Vélez-Rubio et al. 2013). En cada hallazgo, se tomó la posición geográfica, se obtuvieron fotografías y se tomaron las medidas morfométricas, entre ellas el largo curvo de caparazón (LCC). Las zonas de varamiento se ubicaban en el Departamento de Rocha, Uruguay, entre los balnearios de Barra de Valizas

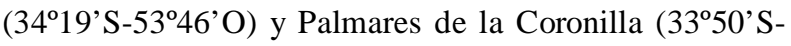
$53^{\circ} 26^{\prime} \mathrm{O}$ ), abarcando aproximadamente $63 \mathrm{~km}$ de costa (Fig.
1). Los epibiontes fueron colectados en su totalidad, mediante un cuidadoso raspado con espátula, bisturí, y cuando fue necesario se utilizaron pinzas. Las muestras se colocaron en frascos de plástico y fueron preservadas en etanol $70 \%$ en el Centro de Investigación y Conservación de Tortugas Marinas de Karumbé, en La Coronilla, Rocha. Para la identificación taxonómica se realizó un análisis morfológico, usando literatura especializada y guías de identificación. Los organismos más pequeños fueron analizados en una lupa marca Boreal T1A. El material analizado fue depositado en el Museo de Historia Nacional de Uruguay.

\section{RESULTADOS Y DisCUSIÓN}

Las tortugas analizadas fueron individuos juveniles, con una longitud media de $\mathrm{LCC} \pm \mathrm{DT}=34,4 \pm 0,75 \mathrm{~cm}(\mathrm{DT}=$ desviación estándar de las medidas del caparazón de los 4 individuos). Se registraron epibiontes correspondientes a 3 grupos taxonómicos: algas, cirripedios e hirudíneos (Tabla 1). Las especies de algas encontradas fueron Sphacelaria sp. e Hincksia mitchelliae, ambas filamentosas pertenecientes a la clase Phaeophyceae. Las especies del género Sphacelaria son comunes en gran parte del océano Atlántico (AlgaeBase 2015) y ha sido previamente descrito como epibionte de la tortuga carey en las costas de Australia (Cribb 1969) y Tanzania (Frazier et al. 1992). Por otro lado, Hincksia mitchelliae ha sido registrada en asociación a la tortuga cabezona en el Atlántico Oriental (Liria 2011). Sin embargo, no se han encontrados reportes previos de esta alga en tortuga carey.
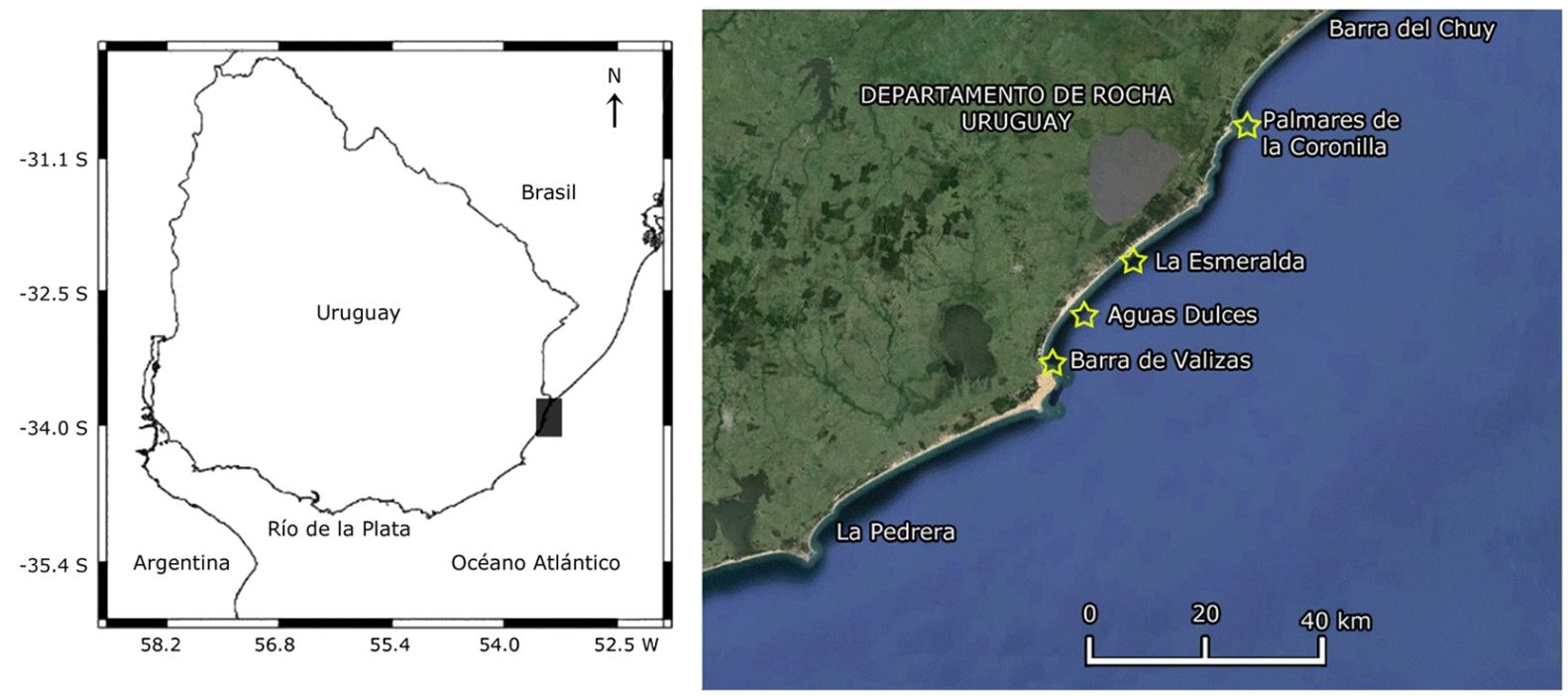

Figura 1. Zonas de varamientos (indicados con una estrella) de 4 ejem plares de tortuga carey, Departamento de Rocha, Uruguay / Stranded area (yellow star) of 4 Hawksbill sea turtles in the coast of Rocha Department, Uruguay 
Las algas filamentosas o calcáreas constituyen la epibiota más común encontrada en la tortuga carey (Schärer 2003), su importancia radica en la capacidad de formar un sustrato capaz de atrapar partículas, proporcionando un microambiente arenoso adecuado para la meiofauna asociada (Schärer 2003).

Los epibiontes invertebrados más frecuentemente hallados fueron los cirripedios (Tabla 1), contabilizándose un total de 377 individuos: Platylepas hexastylos se registró con la mayor abundancia $(n=365)$ en los 4 ejemplares de tortuga carey, mientras que Amphibalanus improvisus y Chelonibia testudinaria sólo se encontraron en una tortuga. Se observó que los balánidos presentes en la tortuga carey fueron mayoritariamente de los géneros Platylepas y Chelonibia, siendo el primero más frecuente en la piel y el segundo en el caparazón (Schärer 2003, Cárdenas-Palomo \& MaldonadoGasca 2005). La especie Amphibalanus improvisus presenta una amplia distribución en todo el mundo y ha sido reportada en el Atlántico Occidental por Rocha (2008). Sin embargo, hasta la fecha no se había descrito como epibionte de la tortuga carey. Los cirripedios han sido reportados como los epibiontes más comunes en varias especies de tortugas marinas (Kitsos et al. 2003, Casale et al. 2012); se los considera organismos macroscópicos pioneros en la colonización puesto que generan sustrato y refugio para otros organismos (Frick et al. 2002). De acuerdo a Frick \& Pfaller (2013), muchos cirripedios son comensales obligados de las tortugas marinas, ya que son encontrados casi exclusivamente en ellas y dependen de la tortuga para su supervivencia. A su vez, estos epibiontes pueden llegar a ser muy perjudiciales para las tortugas marinas: cuando la comunidad es muy grande y alcanza un peso considerable, pueden reducir la flotabilidad, movilidad e hidrodinamismo de la tortuga, incluso pueden traspasar el caparazón completamente y dañar la musculatura del animal (Liria 2011).

El tercer taxón encontrado corresponde al hirudíneo Ozobranchus margoi. Esta especie se alimenta de la sangre de tortugas marinas y habita las costas del ASO (McGowing et al. 2011). El principal hospedador para $O$. margoi es la tortuga cabezona; sin embargo, también hay registros en tortuga verde y carey (McGowing et al. 2011). Además, esta especie se ha reportado en tortugas verdes juveniles en Uruguay (Bevilacqua 2011). Ozobranchus margoi puede llegar a ser muy perjudicial para las tortugas marinas, ya que se ha descrito como vector del virus de la fibropapilomatosis (Orós et al. 1999, McGowing et al. 2011), enfermedad que fue inicialmente descubierta en tortuga verde, pero que se describió posteriormente en tortuga carey (Orós et al. 1999).

La importancia del conocimiento de los epibiontes radica en que pueden proporcionar información indirecta acerca de aspectos biológicos de los quelonios que son difíciles de elucidar, tales como sus rutas migratorias y determinados aspectos de su ciclo de vida (Casale et al. 2012, Domènech et al. 2014). Recopilar información sobre los epibiontes de tortugas marinas ayuda a conocer la dinámica de las diferentes poblaciones; la cantidad y especies de epibiontes pueden dar indicios de las características del área de alimentación y tiempo de permanencia en las mismas, además del estado de salud de las tortugas marinas. El hecho de que estos ejemplares hayan

Tabla 1. Epibiontes de 4 tortugas carey varadas en el Departamento de Rocha, Uruguay, en el 2009 y 2011. NI= total de individuos. LCC= largo curvo del caparazón. * primer reporte para la tortuga carey / Epibionts collected from 4 hawksbill turtles stranded on the coast of Rocha Department, Uruguay, in 2009 and 2011. $\mathrm{Nl}=$ total number of individuals. LLC= curve carapace length. * First report for hawksbill turtles

\begin{tabular}{|c|c|c|c|c|c|}
\hline Fecha & Localidad & Epibionte & NI & $\begin{array}{l}\text { Región } \\
\text { Corporal }\end{array}$ & $\operatorname{LCC}(\mathrm{cm})$ \\
\hline 19-01-09 & Palmares de La Coronilla & $\begin{array}{l}\text { Platylepas hexastylos } \\
\text { Platylepas hexastylos } \\
\text { Ozobranchus margoi }\end{array}$ & $\begin{array}{c}284 \\
71 \\
1\end{array}$ & $\begin{array}{l}\text { Caparazón } \\
\text { Piel } \\
\text { Piel }\end{array}$ & 35,5 \\
\hline 03-03-11 & La Esmeralda & $\begin{array}{l}\text { Platylepas hexastylos } \\
\text { Chelonibia testudinaria }\end{array}$ & $\begin{array}{l}1 \\
1\end{array}$ & $\begin{array}{l}\text { Caparazón } \\
\text { Caparazón }\end{array}$ & 34,6 \\
\hline $07-03-11$ & Aguas Dulces & $\begin{array}{l}\text { Platylepas hexastylos } \\
\text { Sphacelaria sp. } \\
\text { *Hincksia mitchelliae }\end{array}$ & 1 & $\begin{array}{l}\text { Caparazón } \\
\text { Caparazón } \\
\text { Caparazón }\end{array}$ & 33,7 \\
\hline 09-03-11 & Barra de Valizas & $\begin{array}{l}\text { Platylepas hexastylos } \\
\text { *Amphibalanus improvisus }\end{array}$ & $\begin{array}{c}8 \\
11\end{array}$ & $\begin{array}{c}\text { Piel } \\
\text { Caparazón }\end{array}$ & 33,7 \\
\hline
\end{tabular}


sido encontrados varados también podría dar indicios de una mala condición física, lo que a su vez puede incrementar la presencia de epibiontes.

Los antecedentes entregados en el presente estudio pueden contribuir al conocimiento de las relaciones que mantiene la tortuga carey con sus epibiontes. Esta información ayuda a entender que las tortugas marinas contribuyen a la dispersión de muchos invertebrados que crecen sobre ellas, funcionando como verdaderos corredores genéticos gracias a su naturaleza migratoria (Schärer 2003). Es importante que se sigan generando estudios sobre la epibiosis en las tortugas marinas de la región ASO y comenzar a incorporar análisis moleculares, ya que los análisis morfológicos podrían no ser suficiente en algunos casos. Dadas las disminuciones en las poblaciones de tortugas marinas a nivel mundial, se vuelve necesario entender comoéstas se relacionan con su entorno, más aún cuando existen componentes bióticos dentro del mismo que pueden poner en riesgo la salud de las poblaciones actuales.

\section{Agradecimientos}

A Alejandro Fallabrino, Director Ejecutivo de la ONG Karumbé, Uruguay. A todos los voluntarios, técnicos y coordinadores de la temporada 2014-2015. A Pilar MuñozMuga, docente de la Facultad de Ciencias del Mar y de Recursos Naturales de la Universidad de Valparaíso, por su colaboración en la identificación taxonómica de las algas.

\section{LITERATURA CITADA}

AlgaeBase. 2015. World-Wide electronic publication, National University of Ireland, Galway. <http://www.algaebase.org/ search/species/detail/?species_id=Z3186c6e7ac8cf312>

Bevilacqua M. 2011. Ocurrencia de ectoparásitos del género Ozobranchus (Menzies 1791) en individuos juveniles de tortuga verde (Chelonia Mydas) en Cerro Verde, Departamento de Rocha, Uruguay, Tesina de Licenciatura en Biodiversidad, Facultad de Humanidades y Ciencias, Universidad Nacional del Litoral, Santa Fe, 63 pp.

Blumenthal J, F Abreu-Grobois, T Austin, A Broderick, M Bruford, M Coyne, G Ebanks-Petrie, A Formia, P Meylan, A Meylan \& B Godley. 2009. Turtle groups or turtle soup: dispersal patterns of hawksbill turtles in the Caribbean. Molecular Ecology 18: 4841-4853.

Buitrago J \& H Guada. 2002. La tortuga Carey (Eretmochelys imbricata) en Venezuela. Interciencia 27(8): 392-399.

Cárdenas-Palomo N \& A Maldonado-Gasca. 2005. Epibiontes de tortugas de carey juveniles Eretmochelys imbricata en el Santuario de Tortugas Marinas de Río Lagartos, Yucatán, México. CICIMAR Oceánides 20(1,2): 29-35.
Casale P, M D'Addario, D Freggi \& R Argano. 2012. Barnacles (Cirripedia, Thoracica) and associated epibionts from sea turtles in the Central Mediterranean. Crustaceana 85(4-5): 533-549.

Cribb A. 1969. Algae on Hawksbill turtle. Queensland Naturalist 19: 108-109.

Domènech F, F Badillo, J Tomás, J Raga \& FJ Aznar. 2014. Epibiont communities of loggerhead marine turtles (Caretta caretta) in the western Mediterranean: influence of geographic and ecological factors. Journal of the Marine Biological Association of the United Kingdom 95(4): 851-861.

Estrades A, G Vélez-Rubio, M Caraccio \& A Fallabrino. 2013. Exploring southern waters: The presence of Hawksbill turtles in Uruguay. In: Tucker T, L Belskis, A Panagopoulou, A Rees, M Frick, K Williams, R LeRoux \& K Stewart (comp). Proceedings of the Thirty-Third Annual Symposium on Sea Turtle Biology and Conservation. NOAA Technical Memorandum NOAA NMFS-SEFSC 645: 1-263.

Frazier J, E Winston \& C Ruckdeschel. 1992. Epizoan communities on marine turtles. III. Bryozoa. Bulletin of Marine Science 51(1): 1-8.

Frick M \& J Pfaller. 2013. Sea turtle epibiosis. En: Wyneken J, K Lohmann \& J Musick (eds). The biology of sea turtles, 457 pp. CRC Press, Boca Ratón.

Frick M, K Williams, D Veljacic, L Pierrard, J Jackson \& S Knight. 2002. Newly documented epibiont species from nesting loggerhead sea turtles (Caretta caretta) in Georgia, USA. Marine Turtle Newsletter 88: 3-5.

Hernández-Vázquez S \& C Valadez-González. 1998. Observations of the epizoa found on the turtle Lepidochelys olivacea at La Gloria, Jalisco, Mexico. Ciencias Marinas 24(1): 119-125.

Kitsos M, M Christodoulou, S Kalpakis, M Noidou \& A Koukouras. 2003. Cirripedia Thoracica associated with Caretta caretta (Linnaeus, 1758) in the Northern Aegean Sea. Crustaceana 76(4): 403-409.

Liria A. 2011. Ecosistemas errantes: Epibiontes como indicadores biogeográficos de tortugas marinas de Canarias. Tesis doctoral, Facultad de Ciencias del Mar, Universidad de las Palmas de Gran Canaria, Las Palmas de Gran Canaria, 349 pp.

Marcovaldi M, G López, L Soares, A Santos, C Bellini \& P Barata. 2007. Fifteen years of hawksbill sea turtle (Eretmochelys imbricata) nesting in Northern Brazil. Chelonian Conservation and Biology 6(2): 223-228.

McGowin A, T Truong, A Corbett, D Bagley, L Ehrhart, M Bresette, S Weege \& D Clark. 2011. Genetic barcoding of marine leeches (Ozobranchus spp.) from Florida sea turtles and their divergence in host specificity. Molecular Ecology Resources 11:271-278.

Meylan A \& M Donnelly. 1999. Status Justification for Listing the Hawksbill Turtle (Eretmochelys imbricata) as Critically Endangered on the 1996 IUCN Red List of Threatened Animals. Chelonian Conservation and Biology 3(2): 200-224. 
Mortimer J \& M Donnelly. 2008. Marine turtle specialist group 2007 IUCN red list status assessment, hawksbill turtle (Eretmochelys imbricata), $121 \mathrm{pp}$. IUCN/SSC-Marine Turtle Specialist Group. <http://www.widecast.org/ Resources/Docs/MTSG_Assessment_EI_2007.pdf>

Orós J, J Lackovich, E Jacobson, D Brown, A Torrent, S Tucker \& P Klein. 1999. Fibropapilomas cutáneos y fibromas viscerales en una tortuga verde (Chelonia mydas). Revista Española de Herpetología 13: 17-26.

Ortega L \& A Martínez. 2007. Multiannual and seasonal variability of water masses and fronts over the Uruguayan shelf. Journal of Coastal Research 23: 618-629.

Rocha C. 2008. Cirripedia Balanomorpha del estuario del Río Paripe (Isla de Itamaracá, Pernambuco, Brasil). Biota Neotropical 8(3): 31-39.
Schärer M. 2003. A survey of the epibiota of Eretmochelys imbricata (Testudines: Cheloniidae) of Mona Island, Puerto Rico. Revista de Biología Tropical 51(4): 87-90.

UICN. 2014. Eretmochelys imbricata. The IUCN Red List of Threatened Species 2008: e.T8005A12881238. <http:// www.iucnredlist.org/details/8005/0>

Vélez-Rubio G, A Estrades, A Fallabrino \& J Tomás. 2013. Marine turtle threats in Uruguayan waters: insights from 12 years of stranding data. Marine Biology 160(11): 2797-2811.

Recibido el 25 de agosto de 2015 y aceptado el 21 de abril de 2016

Editor: Claudia Bustos D. 